\title{
Synthesis of bis(pyrrolidone-4-carboxylic acid)-based polyamides derived from renewable itaconic acid- application as a compatibilizer in biopolymer blends
}

\author{
Farouk Ayadi ${ }^{1}$, Saskia Mamzed ${ }^{2}$, Charles Portella ${ }^{2}$ and Patrice Dole ${ }^{1}$ \\ The properties and morphologies of blends of poly(butylene succinate) (PBS) and glycerol-plasticized thermoplastic starch \\ (TPS) were investigated. Bis-pyrrolidone-type monomers (obtained from itaconic acid) with various sizes of alkylidene spacers \\ were synthesized and polycondensed with various diamines. The resultant polycondensates were used as compatibilizers to \\ improve interfacial adhesion in PBS/starch blends. The presence of long hydrophobic $-\mathrm{CH}_{2}-$ sequences in the compatibilizers \\ improved the elongation-at-break during tensile tests. The enhanced compatibilization induced by these copolymers is \\ presumably because of the hydrophobic interaction with PBS and the hydrophilic hydrogen bonding with starch. \\ The uncompatibilized TPS/PBS exhibited a dispersed morphology with blends of low- and high-starch content, whereas \\ the compatibilized blends presented a co-continuous phase.
}

Polymer Journal (2013) 45, 766-774; doi:10.1038/pj.2012.206; published online 5 December 2012

Keywords: itaconic acid; mechanical properties; morphology; poly(butylene succinate) (PBS); pyrrolidone; thermoplastic starch (TPS)

\section{INTRODUCTION}

The widespread use of synthetic nondegradable polymers has had a severe environmental impact, and one solution to this problem is to use biodegradable polymers. Thermoplastic starch (TPS) is one of the most studied materials because of its renewable origin, biodegradability and low cost. However, plasticized starch cannot be used to replace conventional plastics because of its poor mechanical properties and water sensitivity. ${ }^{1,2}$

To overcome these shortcomings, inexpensive TPS has been blended with biodegradable polyester to retain most of the physical properties of the polyesters. A considerable amount of research has been conducted on the development of starch blends with polyester polymers, ${ }^{3}$ such as polylactide (PLA)-starch, ${ }^{4-7}$ polycaprolactone (PCL)-starch ${ }^{8-10}$ and poly(butylene succinate) (PBS)-starch. ${ }^{11-13}$ These blends present several advantages. ${ }^{14}$ The composition of the blends can be adjusted to obtain properties that meet the needs of the application, and the blending process is inexpensive compared with the cost of developing new synthetic materials.

Unfortunately, the mechanical properties of these blends tend to diminish considerably as the plasticized starch fraction increases. The properties of plasticized starch/polymer blends can be greatly improved by using a compatibilizer. ${ }^{15,16}$
Studies of starch blends with aliphatic polyesters (PCL, PBS, PHBV) showed, in all cases, that a polymer with acceptable properties could be produced with only a moderate amount of starch. The compatibility between the starch and the aliphatic polyester is commonly improved by incorporating anhydride functionalized compatibilizers into the polyester backbone. Only a small amount of the compatibilizer is required to produce a blend that has tensile strength similar to that of synthetic polyester. ${ }^{17}$

Itaconic acid (IA), which is produced by fermentation from hexoses, is an interesting renewable building block for diverse applications. ${ }^{18}$ This acid has also been recognized as one of the 'Top Value Added Chemicals' from carbohydrate biomass. ${ }^{19}$ The primary interest in IA stems from the diacid character of its acrylic functionality. Thus, itaconic acid reacts with primary amines in a Michael additioncyclocondensation sequence to produce carboxylic acid-functionalized pyrrolidones. ${ }^{20}$ Polyamides with various sizes of alkylidene spacers can be synthesized by the polycondensation of these diacids with different diamines. The resulting amphiphilic polycondensates should exhibit an adjustable hydrophilic-hydrophobic balance.

In this paper, we report on the improved interfacial adhesion between PBS and plasticized starch obtained using polycondensate compatibilizers synthesized from itaconic acid.

${ }^{1}$ Equipe Transformations Physico-chimiques des matériaux lignocellulosiques, UMR FARE, INRA-URCA, Reims, France and ${ }^{2}$ Université de Reims-Champagne-Ardenne, Institut de Chimie Moléculaire de Reims, UMR CNRS 6229, UFR Sciences Exactes et Naturelles, Reims, France

Correspondence: Dr F Ayadi, Equipe Transformations Physico-chimiques des matériaux lignocellulosiques, UMR FARE, INRA-URCA, Campus Moulin de la Housse, Bât 18, Reims 51687, France.

E-mail: Farouk.ayadi@iit.it

Received 8 March 2012; revised 12 September 2012; accepted 19 September 2012; published online 5 December 2012 


\section{MATERIAL AND METHODS}

\section{Preparation and properties of bis(pyrrolidone)-based compatibilizers}

General methods and reagents. NMR spectra were recorded in $\mathrm{D}_{2} \mathrm{O}$ or DMSO with a Bruker AC 250 (Wissembourg, France) at frequencies of 250 and $62.9 \mathrm{MHz}$ for ${ }^{1} \mathrm{H}$ and ${ }^{13} \mathrm{C}$ NMR, respectively. The chemical shifts $(\delta)$ are reported in p.p.m. relative to TMS.

The molecular weight distribution of the polycondensates was determined by size-exclusion chromatography on a customized Waters HPLC system that was equipped with a Waters 600 HPLC pump, a Waters (Milford, MA, USA) 710plus automated injector, a Jasco (Jasco International Co., Ltd., Tokyo, Japan) CO-965 column oven and a Waters 410 Refractive Index detector. The analyses were performed on a system of three columns in series (SB $805 \mathrm{HQ}$, SB $803 \mathrm{HQ}$ and SB 802.5 HQ) (Shodex, Germany). DMSO was used as the mobile phase at a flow rate of $1 \mathrm{ml} \mathrm{min}{ }^{-1}$. The temperature of the column oven was set at $35^{\circ} \mathrm{C}$. Processing of all of the chromatographic data was performed using version 2.0 of the Empower software package (Waters) customized with a GPC license. Analyses were performed on samples dissolved in DMSO at $\sim 1 \mathrm{gl}^{-1}$. An injection volume of $50 \mu \mathrm{l}$ was eluted at a rate of $1 \mathrm{mlmin}^{-1}$ during an acquisition period of $45 \mathrm{~min}$. The column porosity allowed good separation of molecules ranging from $8500-4000000 \mathrm{~g} \mathrm{~mol}^{-1}$. The instrument was calibrated using monodisperse pullulan standards to calculate the $\mathrm{Mw}$ and $\mathrm{Mn}$.

Differential scanning calorimetry experiments were conducted using a TA 2920 DSC instrument (TA Instruments Ltd., New Castle, DE, USA). Sealed pans were used to encapsulate typically $20 \mathrm{mg}$ of the compatibilizer. For accurate heat capacity measurements, the sample and reference pans were matched. The DSC instrument was calibrated from the melting temperature and enthalpy of the standard material indium. The samples were accurately weighed in an aluminum pan and hermetically sealed. An empty pan was used as the reference.

Itaconic acid and p-toluenesulfonic acid were supplied by Sigma Aldrich (Lyon, France), ethylenediamine (extra pure) was purchased from Acros (Geel, Belgium) and 1,6-diaminoethane (98\%) was supplied by Alfa Aesar (Schiltigheim, France).

Synthesis and polycondensation of bis(pyrrolidones). The starting bis-(pyrrolidones) were prepared by heating a solution of itaconic acid $(60 \mathrm{~g}, 0.51 \mathrm{~mol})$ and the corresponding diamine ( 0.5 equiv.) in water $(500 \mathrm{ml})$ to reflux for $16 \mathrm{~h}$. The water was removed using a Dean Stark apparatus (University of Reims, Gaffer-service, Reims, France) to yield an oil, which was then triturated with methanol to yield the product as a white solid. Several successive trituration-filtration sequences may be necessary to recover the product with a high yield. However, the addition of a trace amount of AcOEt may favor the crystallization of the product.

Bis-1,2-(4-carboxypyrrolidin-2-on-1-yl)ethane. Yield: $115.9 \mathrm{~g}, 89 \% . F=239$ $243{ }^{\circ} \mathrm{C} .{ }^{1} \mathrm{H}$ NMR $\left(\mathrm{D}_{2} \mathrm{O}\right): \delta$ (p.p.m.) $2.95(\mathrm{~m}, 4 \mathrm{H}), 3.63(\mathrm{~m}, 2 \mathrm{H}), 3.78(\mathrm{~m}, 4$ $\mathrm{H}), 4.01(\mathrm{~m}, 4 \mathrm{H}) .{ }^{13} \mathrm{C}$ NMR $\left(\mathrm{D}_{2} \mathrm{O}\right): \delta$ (p.p.m. $) 34.2\left(2 \mathrm{CH}_{2}\right), 36.1(2 \mathrm{CH})$, $39.9\left(2 \mathrm{CH}_{2}\right), 49.9\left(2 \mathrm{CH}_{2}\right), 176.5(2 \mathrm{CO}), 177.3(2 \mathrm{CO})$.

Bis-1,6-(4-carboxypyrrolidin-2-on-1-yl)hexane. Yield: $132.1 \mathrm{~g}, 83 \% . F=245-$ $247^{\circ} \mathrm{C} .{ }^{1} \mathrm{H}$ NMR (DMSO): $\delta$ (p.p.m.) $1.06(\mathrm{~m}, 4 \mathrm{H}), 1.28(\mathrm{~m}, 4 \mathrm{H}), 2.31$ (m, $4 \mathrm{H}), 3.03$ (m, $6 \mathrm{H}$ ), 3.39 (m, $4 \mathrm{H}) .{ }^{13} \mathrm{C}$ NMR (DMSO): $\delta$ (p.p.m.) 26.0
(2 $\left.\mathrm{CH}_{2}\right), 26.8\left(2 \mathrm{CH}_{2}\right), 34.1\left(2 \mathrm{CH}_{2}\right), 35.9(2 \mathrm{CH}), 41.6\left(2 \mathrm{C} \mathrm{CH}_{2}\right), 48.8$ $\left(2 \mathrm{CH}_{2}\right), 172.3(2 \mathrm{CO}), 175.1(2 \mathrm{CO})$.

The polycondensates were prepared by heating bis(pyrrolidone) and the diamine ( 1 equiv.) in the presence of a p-toluenesulfonic acid as a catalyst (PTSA) (0.03 equiv.) in a small amount of water for $1 \mathrm{~h}$ at $100{ }^{\circ} \mathrm{C}$ under argon. The mixture was then heated to $170^{\circ} \mathrm{C}$ for 3 days under a continuous vacuum to remove the water. The reaction schemes and simplified nomenclature of the products are depicted in Figure 1.

The compatibilizers were stored for 5 days in glass hygrostats at $20^{\circ} \mathrm{C}$ at different relative humidities $(0,13,33$ or $50 \% \mathrm{RH})$ before being placed in pressure-tight cells. The glass transition temperatures of these compatibilizers were determined using DSC. In fact, after an initial scan from -50 to $200{ }^{\circ} \mathrm{C}$ at a heating rate of $5^{\circ} \mathrm{C} \mathrm{min}^{-1}$ to delete any thermal events that occur in the compatibilizer, the samples were rapidly cooled to $-50^{\circ} \mathrm{C}\left(25^{\circ} \mathrm{C} \mathrm{min}{ }^{-1}\right)$. The measurements were performed during a second scan at a rate of $10^{\circ} \mathrm{Cmin}^{-1}$ from -50 to $200{ }^{\circ} \mathrm{C}$. The glass transition temperature, $\mathrm{Tg}^{1 / 2}$, which corresponds to a half variation in calorific capacity during the transition, was determined graphically. ${ }^{21}$ The procedure was similar for all samples, regardless of their compositions.

\section{Blends preparation and processing conditions}

Native corn starch, which contained $~ 74 \%$ amylopectin, $26 \%$ amylose and $0.2 \%$ proteins, and had a moisture content of $12 \% \mathrm{w} / \mathrm{w}$, was purchased from Sigma Aldrich (Germany). Glycerol $\left(<0.5 \% \mathrm{H}_{2} \mathrm{O}\right)$ was obtained from Carlo Erba reactif (Italy).

Polybutylene succinate (PBS) was supplied by the Mitsubishi Chemical Corporation (Tokyo, Japan)(GSPLA as a reference). The water content of the starch was considered in all of the experiments.

All of the products were used without any further purification.

The processing began with the preparation of the blend (starch with $25 \%$ of glycerol). Corn starch (containing 12\% of moisture) was weighed and introduced into a turbo-mixer. After the addition of glycerol, the two compounds were mixed at a high speed (2000 r.p.m.).

The starch-glycerol blend was extruded on a single screw extruder (SCAMIA S 2032, France) equipped with a conical-shaped element (see description and figures in Onteniente et $a l^{22}$ ). This design is suitable for high shearing. The extruder had two heating zones situated around the head $\left(115^{\circ} \mathrm{C}\right)$ and at the die $\left(95^{\circ} \mathrm{C}\right)$. The extruded blends were granulated.

These different plastic granules were premixed with PBS at ratios of 30:70 (starch-to-PBS) (SPB30), 40:60 (SPB40), 60:40 (SPB60) and 70:30 (SPB70) at room conditions. The compatibilizer (1\%) was added to each of the resulting TPS-PBS pellets. Each mixture was manually fed into the extruder. The resulting blends were granulated then extruded again to improve the dispersion of the compatibilizer within the matrix. The last extrusion was performed at 65 r.p.m. in a three-zone single-screw extruder (Scamex, France), which was heated to $120^{\circ} \mathrm{C}$ in all zones. The screw was 220-mm long, and the $\mathrm{L} / \mathrm{D}$ ratio was equal to 11 . A flat die (heated to $110^{\circ} \mathrm{C}$ ) was used to obtain the materials in a ribbon form.

Specimen preparation and mechanical tests. The pellets obtained by extrusion were transformed into tensile test samples (ISO 527-1 BA), which were $\sim 1$-mm thick, using a cookie cutter and press. The samples were hydrated in

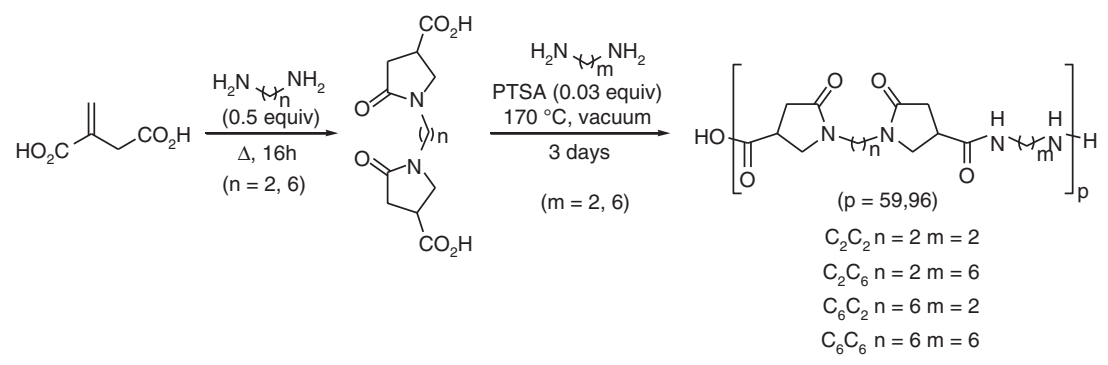

Figure 1 Reaction scheme and nomenclature of the synthesized compatibilizers. 
$50 \%$ RH in chambers that were maintained at $20^{\circ} \mathrm{C}$. The tensile measurements were performed using a tensile test machine (GT-TEST 108, Strasbourg, France) equipped with a load cell of $2 \mathrm{kN}$. The strength, modulus and elongation-at-break were measured using a crosshead speed of $10 \mathrm{~mm} \mathrm{~min}^{-1}$ under constant conditions $\left(50 \% \mathrm{RH}, 20^{\circ} \mathrm{C}\right)$ in an environmentally controlled room. Averages were obtained for ten samples.

Glass transition detection. Mechanical relaxation measurements were performed on a Dynamic Mechanical Analyzer (DMTA MK IV, TA Instruments Ltd.). Molded materials were obtained using an injection molding machine (DSM Xplore, Geleen, The Netherlands), (pellets obtained by extrusion processing were transformed into strip samples (ISO 527-2 5A) (strips, $22 \cdot 10 \cdot 1 \mathrm{~mm}^{3}$ )) and were hydrated for at least 10 days at $50 \% \mathrm{RH}$ (relative humidity) in chambers controlled at $20^{\circ} \mathrm{C}$. The samples were measured in the dual cantilever mode with a vibration frequency of $1 \mathrm{~Hz}$, a strain of $0.1 \%$ and a heating rate of $2{ }^{\circ} \mathrm{Cmin}^{-1}$.

The temperature of the $\alpha$ relaxation was determined at the maximum of tan $\delta=\mathrm{E}^{\prime \prime} / \mathrm{E}^{\prime}$, which corresponds to the molecular mobility associated with the calorimetric glass transition of the material.

\section{Blend morphology}

Investigation of SEM pictures. Scanning electron microscopy micrographs of the different blends were obtained using a scanning electron microscope (model SEM XL 30 from PHILIPS, Eindhoven, The Netherlands). Crosssections of the extruded samples were obtained after fracturing in liquid nitrogen, and then they were treated with methylene chloride (DCM) for 15 min to highlight the dispersion of starch in the PBS. These samples were fixed on carbon-adhesive bearings and coated with gold-palladium, using a plasma sputtering apparatus (model SCD 040 from BALZERS, Weisbaden, Germany) before the SEM investigation.

Investigation by confocal microscopy. The morphology of each TPS-PBScompatibilizer blend was examined using a Leica TCS SP2 confocal microscope with a $\times 63$ magnifying oil objective (numerical aperture 1.4). The samples in film form were placed on the microscope stage, and the acquired picture was magnified twice. When the fluorescent compatibilizer was detected, the appropriate software was used to select the region of interest. A $20 \mathrm{~mW}$ Helium-Neon laser was used at full intensity, and fluorescence recovery was monitored at the lowest possible intensity.

\section{RESULTS AND DISCUSSION}

\section{Synthesis and analysis of compatibilizers}

Different conditions were tested to optimize the synthesis of polyamides with the highest possible molecular weight. The different polymers were synthesized, according to the previously described procedure, and the product was directly isolated as a transparent, yellow, brittle solid, which was then analyzed using ${ }^{1} \mathrm{H}$ NMR and sizeexclusion chromatography.

The suspension of bis(pyrrolidone) in water dissolved immediately after the amine was added to the solution. The aqueous solvent, which helps the formation of a polyamide salt during the first step of the reaction, was removed when the reaction was placed under vacuum. Although no important differences were observed between the different acid catalysts tested (PTSA, $\mathrm{H}_{3} \mathrm{BO}_{3}$ and $\mathrm{H}_{2} \mathrm{SO}_{4}$ ), a slightly higher conversion was obtained using PTSA.

Various reaction temperatures $\left(100,150,170\right.$ and $\left.200^{\circ} \mathrm{C}\right)$ were studied. As expected, the molecular weight increased with the reaction temperature, but some degradation was observed at $200^{\circ} \mathrm{C}$. A kinetic study of the synthesis of the $\mathrm{C}_{2} \mathrm{C}_{2}$ polyamide was therefore conducted to determine the reaction time that would lead to a polymer with sufficient molecular weight but without degradation of the compounds. In this work, the minimum molecular weight for an optimum interpenetration of the two phases was arbitrarily targeted as $20000 \mathrm{~g} \mathrm{~mol}^{-1}$. However, the molecular weight continuously increased with the reaction time, from $\sim 2000 \mathrm{~g} \mathrm{~mol}^{-1}$ after $3 \mathrm{~h}$, and reached a plateau at approximately $30000 \mathrm{~g} \mathrm{~mol}^{-1}$ after $72 \mathrm{~h}$ at $170{ }^{\circ} \mathrm{C}$. A further increase of the reaction time led to coloring of the product, which was most likely owing to degradation. Four different polyamides were prepared by condensing a combination of 1,2-bis(4carboxypyrrolidin-2-on-1-yl)ethane or 1,2-bis(4-carboxypyrrolidin-2on-1-yl)hexane with ethylene diamine or hexylene diamine at $170{ }^{\circ} \mathrm{C}$ for $72 \mathrm{~h}$ in the presence of a catalytic amount of PTSA (Figure 1).

${ }^{1} \mathrm{H}$ NMR analysis of the polycondensates is exemplified with the $\mathrm{C}_{2} \mathrm{C}_{2}$ compound: a signal at 3.21 p.p.m. corresponding to the methylene group $\mathrm{CH}_{2}-\mathrm{NH}-\mathrm{CO}$ and a signal at 8.21 p.p.m. corresponding to the $\mathrm{NH}$ group indicated the formation of the amide. The NMR result also confirms the complete consumption of the starting diamine. Similar spectra were observed for all of the compounds.

Regarding the polymer size, the best result was obtained for $\mathrm{C}_{2} \mathrm{C}_{2}$ $(\mathrm{Mw}=31000, \mathrm{Ip}=1.8, \mathrm{DPn}=96)$. As expected, similar results were obtained for the $\mathrm{C}_{2} \mathrm{C}_{6}(\mathrm{Mw}=24100, \mathrm{Ip}=1.96, \mathrm{DPn}=64)$ and $\mathrm{C}_{6} \mathrm{C}_{2}$ $(\mathrm{Mw}=25200, \mathrm{Ip}=2.12, \mathrm{DPn}=67)$ polyamides. The lowest molecular weight was obtained for $\mathrm{C}_{6} \mathrm{C}_{6} \quad(\mathrm{Mw}=23200, \quad \mathrm{Ip}=2.06$, $\mathrm{DPn}=59$ ). As expected, the molecular weights of the polyamides decreased as the monomer size increased.

\section{Thermal properties of the synthesized compatibilizers}

The glass transition temperatures of the synthesized polymers decreased as the water content increased (Table 1). The samples exhibited hydrophilic behavior. This result could be explained by the presence of amide groups, which are strong hydrogen-bonding groups and preferentially form hydrogen bonds with water. ${ }^{23}$ Indeed, among the four hydrophilic polymers studied, $\mathrm{C}_{2} \mathrm{C}_{2}$ exhibited considerable sensitivity to water because its $\mathrm{Tg}$ varied from $-15^{\circ} \mathrm{C}$ at $50 \% \mathrm{RH}$ to $124^{\circ} \mathrm{C}$ at $0 \% \mathrm{RH}$. This behavior was attributed to its smaller hydrophobic $\mathrm{CH}_{2}$ sequences compared with the other synthesized polymers.

Not surprisingly, the polycondensate $\mathrm{C}_{6} \mathrm{C}_{6}$ was less sensitive to water and had $\mathrm{Tg}$ values ranging from 29 to $48^{\circ} \mathrm{C}$. We have thus, according to these DSC analyses, synthesized a variety of polycondensates with different sensitivities to water. This property of adjustable hydrophilicity in a compatibilizer is of great interest.

\section{Mechanical and thermal properties of the TPS/PBS blends}

Mechanical properties. Figure 2 presents the elongation-at-break, tensile strength and modulus for PBS as a function of TPS concentration. All of the systems were systematically characterized at $50 \% \mathrm{RH}$ and $20^{\circ} \mathrm{C}$. In all cases, the TPS contained $25 \%$ glycerol.

The strain-at-break of the PBS composites increased with increasing TPS content (Figure 2a). The low strain-at-break of PBS is explained by the chosen characterization procedure: the tensile test specimens were prepared using a cookie cutter to facilitate the characterization of defaults in the material. Indeed, samples obtained

Table 1 Glass transition values of the synthesized polycondensates obtained at different relative humidities

\begin{tabular}{lcccr}
\hline & \multicolumn{4}{c}{ Glass transition temperature $\left({ }^{\circ} \mathrm{C}\right)$} \\
\cline { 2 - 5 } Polycondensate & $0 \% R H$ & $13 \% R H$ & $33 \% R H$ & $50 \% R H$ \\
\hline C2C2 & 124 & 98 & 38 & -15 \\
C2C6 & 103 & 85 & 69 & 64 \\
C6C2 & 98 & 70 & 51 & 45 \\
C6C6 & 48 & 37 & 34 & 29 \\
\hline
\end{tabular}



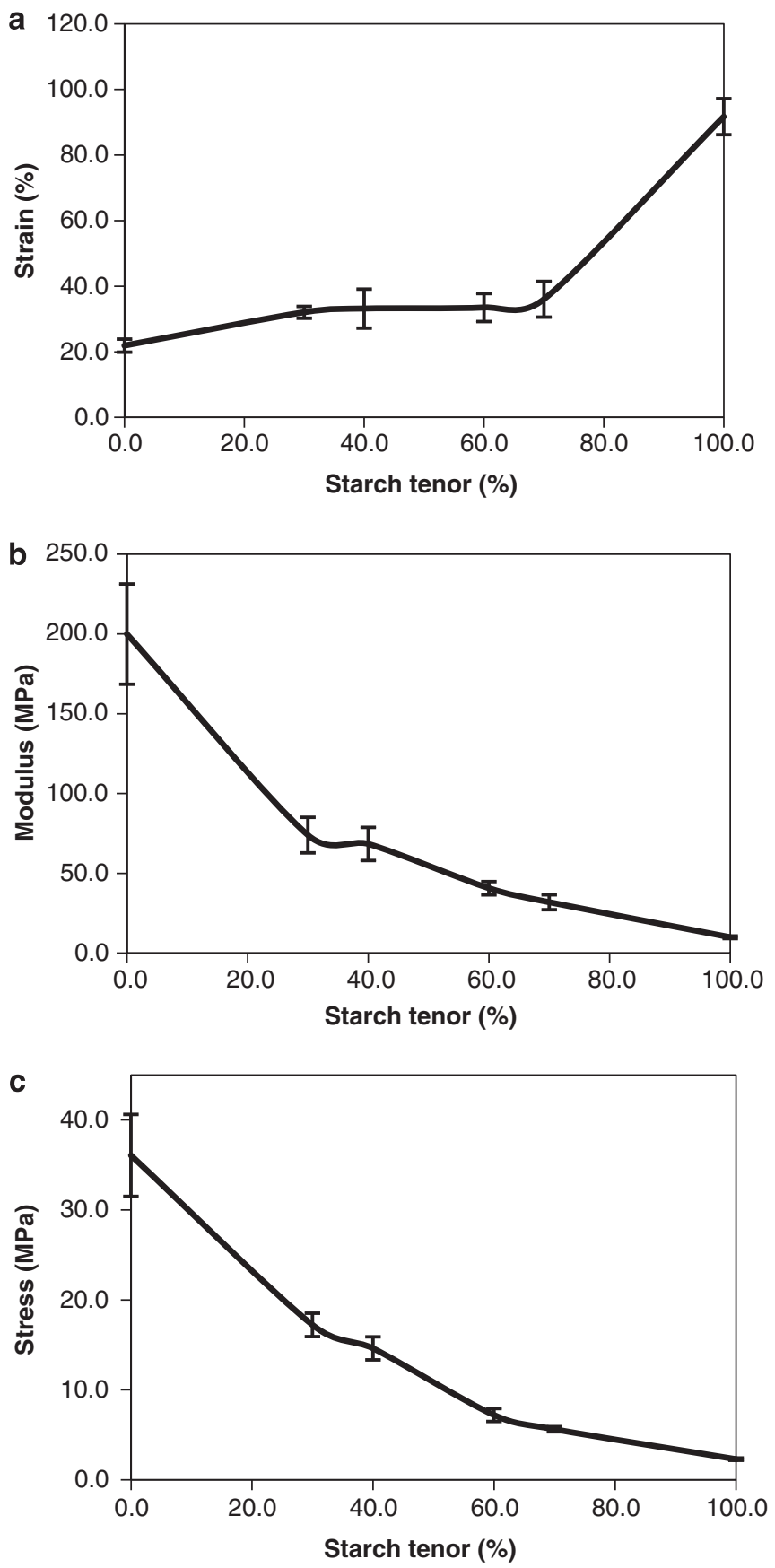

Figure 2 Effect of starch content on (a) the strain, (b) modulus and (c) stress of the starch-poly(butylene succinate) (PBS) blends.

using cutting procedures are sensitive to fracture propagation. As PBS is known to be fragile to fracture propagation, low strain-at-break values were obtained for the pure material. In contrast, the plasticized starch containing $25 \%$ glycerol exhibited plastic behavior, and it was characterized by a higher strain-at-break.

The strain of the PBS/TPS mixtures varied very slightly, increasing from $32 \%$ for SPB30 to $36 \%$ for SPB70, whereas the values for the pure component were 22 and $92 \%$ for PBS and starch, respectively. This behavior suggests that the mechanical properties were related to interfacial defaults between PBS and starch, which has already been reported for TPS/polyester blends. ${ }^{24-27}$

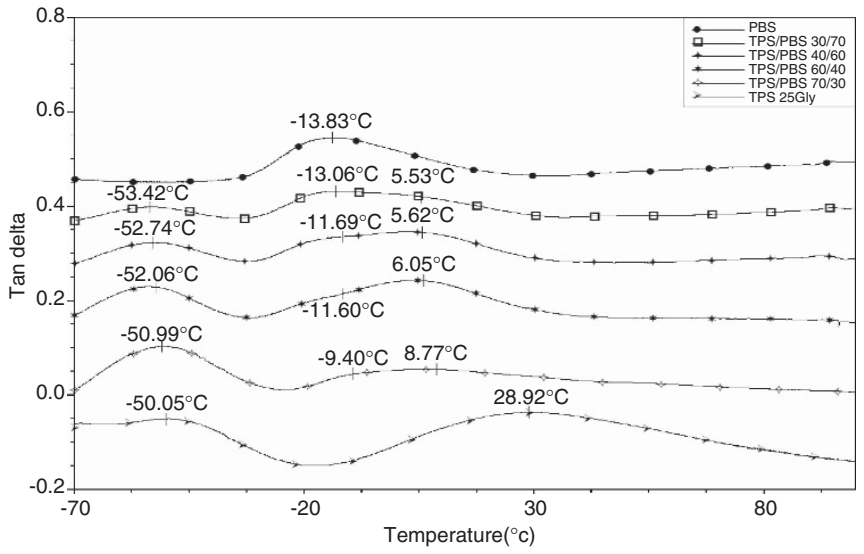

Figure 3 Glass transition values measured with a dynamic mechanical analyzer (DMA) of starch and poly(butylene succinate) (PBS) blends with starch/PBS ratios of 0:100, 30:70, 40:60, 60:40, 70:30 and 100:0.

The tensile modulus progressively decreased with the addition of TPS, which had a much lower intrinsic modulus at the glycerol concentration used here (25\%) (Figure 2b). Furthermore, as the starch content increased to $70 \%$, the modulus of the blends decreased because of the increased content of the discontinuous PBS phase. The strength-at-break (that is, stress) of TPS-PBS was shown to decrease with the increasing amount of starch (Figure 2c), as already observed by other investigators. ${ }^{12,13,28}$

The mechanical properties of the PBS-TPS blends exhibited three different zones: (i) below 30\% starch content, the starch acted as filler in the continuous PBS matrix. As the blends were subjected to an external tensile load, the PBS matrix was the primary load-bearing phase. (ii) When the starch content in the TPS-PBS blends was between 30 and $70 \%$, the mechanical properties varied only slightly. (iii) Above $70 \%$ starch content, the PBS matrix became discontinuous, and the effective cross-section area of the continuous phase was reduced, which resulted in decreased stress.

Dynamic mechanical properties. The most commonly used method to measure the miscibility in polymer-polymer blends or partialphase mixing in such blends is by comparing the $\alpha$ relaxation of the blend with that of the unblended constituents. ${ }^{29-31}$

Figure 3 shows the $\tan \delta$ peaks for 100\% PBS, 100\% starch and starch-PBS composites. The following salient features are apparent:

(i) Presence of two $\alpha$-transitions in the TPS-PBS composites, which correspond to a starch-rich phase and a PBS-rich phase and suggests that the compatibility between the two components is limited.

(ii) Presence of a $\beta$-transition in all samples, except pure PBS. Such $\beta$-transitions were also observed in starch-glycerol-water systems within a similar range of temperatures. ${ }^{24,32,33}$

(iii) Decrease in the $(\tan \delta)_{\max }$ of PBS and an increase in the $(\tan \delta)_{\max }$ of the starch peaks.

(iv) Changes in the $\alpha$-transition temperature of the PBS and starch fractions.

In fact, the $\alpha$-transition of PBS slightly increased with increasing starch content and was observed to range from -13.83 to $-9.40^{\circ} \mathrm{C}$ for PBS and SPB70, respectively. In contrast, the $\alpha$-transition of TPS considerably decreased from $28.92^{\circ} \mathrm{C}$ for pure TPS to $8.77^{\circ} \mathrm{C}$ for 

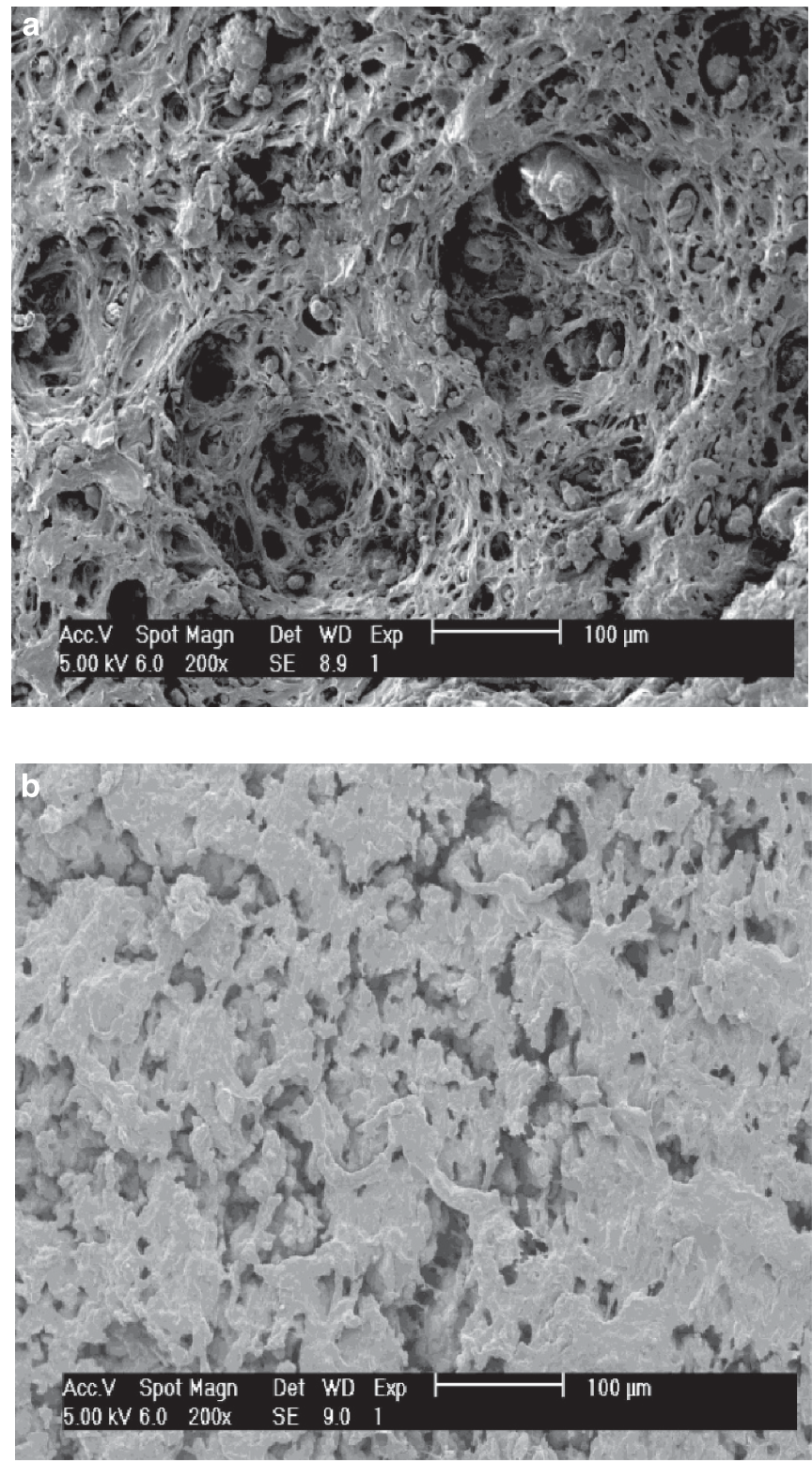

Figure 4 Scanning electron microscope (SEM) pictures of the extruded thermoplastic starch (TPS)/poly(butylene succinate) PBS blend: (a) SPB30 and (b) SPB70.

SPB70 then decreased slightly for all TPS-PBS composites as the PBS content increased.

The $\beta$-transition exhibited a very slight shift to lower temperatures as the amount of PBS increased. This shift could be because of some solubilization of glycerol in PBS. As the $\beta$-transition of the plasticized starch increases with the glycerol content, this negative shift of the peak shows that a part of the glycerol migrates in the PBS phase.

Morphology. The mechanical properties and the morphologies of polymer blends are known to be strongly inter-related. The morphology is of paramount importance; however, in the case of polymer incompatibility, which leads to the development of a two-phase material. Most starch-based plastics have been unable to replace conventional petrochemical polymers because of phase inversion at high-starch contents and the appearance of a continuous TPS phase with poor mechanical properties. ${ }^{24,26,30,34}$
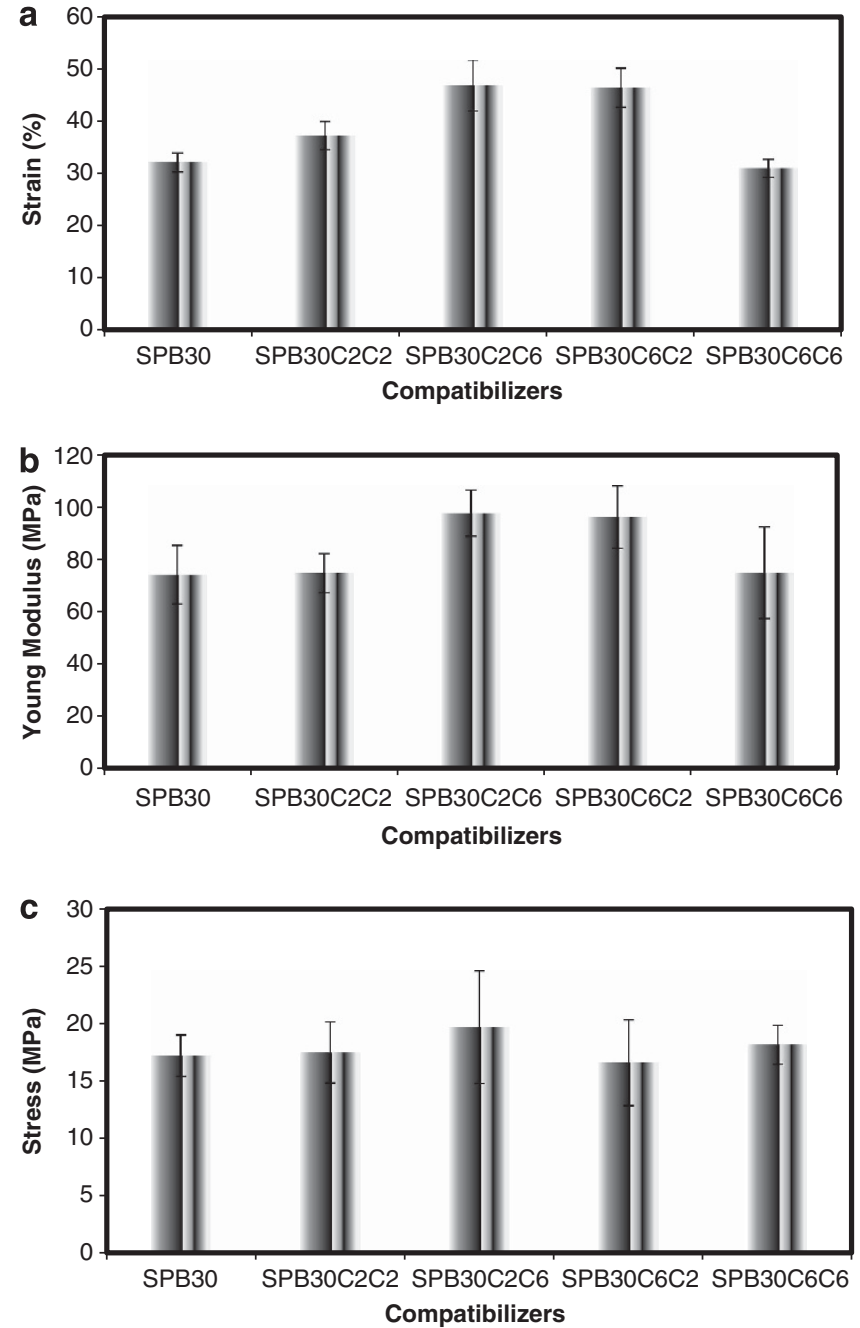

Figure 5 Effect of synthesized compatibilizers on (a) the strain, (b) modulus and (c) stress of SPB30.

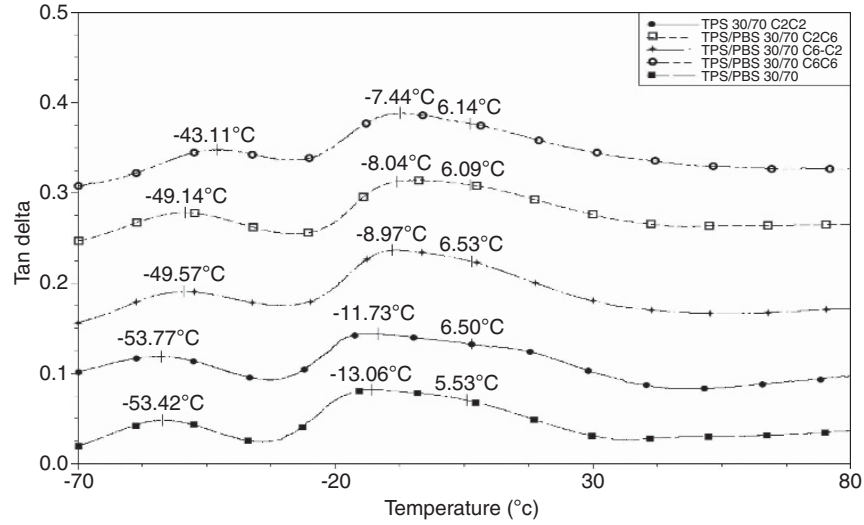

Figure 6 Effect of synthesized compatibilizers on the glass transition values of SPB30 measured with a dynamic mechanical analyzer (DMA). 
The morphology of the fractured TPS-PBS blends is shown in Figure 4. The PBS phase was extracted with dichloromethane for $15 \mathrm{~min}$ to improve the contrast in the micrographs of the blends.

The morphology of a blend with relatively high-starch content (SPB70) was compared with that of a blend with low-starch content (SPB30). As shown in Figure 4a (SPB30), a clear edge and cavity can be observed between the starch granules and the PBS matrix. The starch granules exhibited a broad-size distribution, and some of them were detached from the PBS matrix. The morphology of the highstarch content blend (Figure 4b) still exhibits small cavities but no starch nodule because of the lower PBS content. These results indicate the presence of a dispersed nodular morphology in blends with lowor high-starch contents.

This morphology is typical of incompatible blends and results in poor mechanical properties.
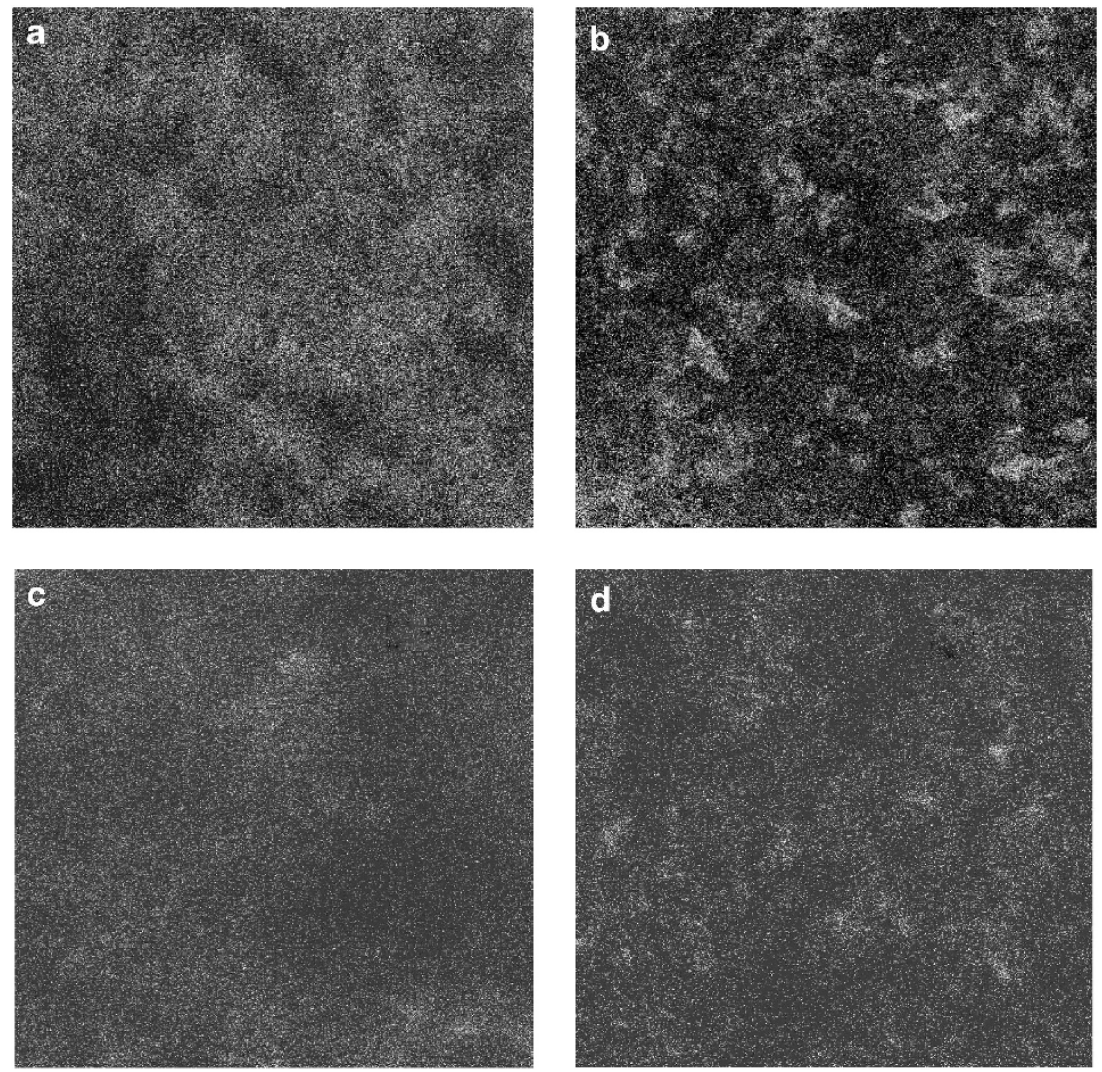

Mechanical and thermal properties. All of the synthesized compatibilizers were tested with the SPB30 blend.

Figure 5 shows the tensile properties of SPB30 with different compatibilizers. The addition of $1 \% \mathrm{C}_{2} \mathrm{C}_{2}$ resulted in little increase of the elongation-at-break and stress, and a slight increase of the

As noted above, the starch $\alpha$-transition in the TPS-PBS blend exhibited a considerable shift at lower temperatures, which indicates some interfacial interaction between the two polymers. This interaction might be because of hydrogen bonding between the starch hydroxyl groups and the polyester. Furthermore, the compatibility of TPS and PBS could be improved by adding the previously synthesized compatibilizer.
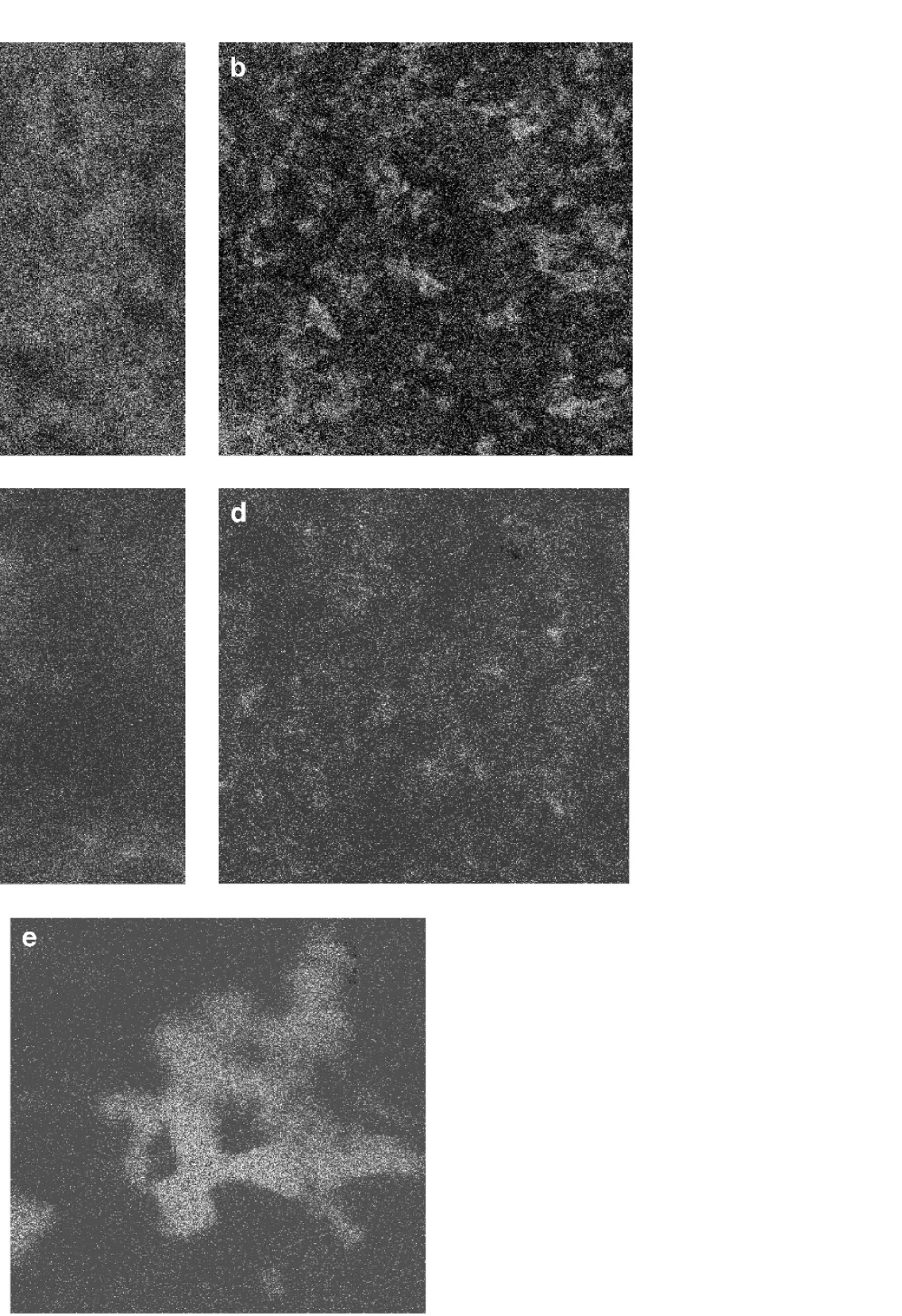

Figure 7 Effect of synthesized compatibilizers on the morphologies of the TPS/PBS blends using confocal microscopy: (a) SPB70- $\mathrm{C}_{2} \mathrm{C}_{2}$, (b) SPB30- $\mathrm{C}_{2} \mathrm{C}_{2}$, (c) SPB30- $\mathrm{C}_{2} \mathrm{C}_{6}$, (d) SPB30- $\mathrm{C}_{6} \mathrm{C}_{2}$ and (e) SPB30- $\mathrm{C}_{6} \mathrm{C}_{6}$. A full color version of this figure is available at Polymer Journal online. 
modulus. Only a $16 \%$ improvement of strain compared with that of a pure blend was observed with the $\mathrm{C}_{2} \mathrm{C}_{2}$ compatibilizer.

A $45 \%$ improvement of the elongation-at-break and a $30 \%$ improvement of the Young's modulus were observed when $\mathrm{C}_{2} \mathrm{C}_{6}$ and $\mathrm{C}_{6} \mathrm{C}_{2}$ were used as compatibilizers, whereas the addition of $\mathrm{C}_{6} \mathrm{C}_{6}$ resulted in a considerable decrease of strain. In fact, the presence of insoluble components in the SPB30- $\mathrm{C}_{6} \mathrm{C}_{6}$ composite, which act as flaws in the matrix and result in poor mechanical properties, were apparent from simple visual observation. The mechanical properties of these blends appeared to depend not only on the mechanical properties of each component but also on the interfacial properties and were therefore directly related to the compatibilization.

The DMA traces of $\tan \delta$ for the SPB30-compatibilizer blends (Figure 6) show three transitions. The lowest transition at $\sim-53.42$ $-43.11^{\circ} \mathrm{C}$ corresponds to the $\beta$-transition of starch, and the $\alpha$ transition is between 5.53 and $6.14{ }^{\circ} \mathrm{C}$. The second transition corresponds to the $\alpha$-transition of PBS, which is generally between -13.06 and $-7.44^{\circ} \mathrm{C}$.

After adding the compatibilizer, the peak for the $\alpha$-transition of the PBS in the compatibilized blends was observed to shift toward higher temperatures, whereas the $\alpha$-transition of starch remained constant.

The addition of $1 \% \mathrm{C}_{2} \mathrm{C}_{2}$ had no effect on glass transition, whereas $\mathrm{C}_{2} \mathrm{C}_{6}, \mathrm{C}_{6} \mathrm{C}_{2}$ and $\mathrm{C}_{6} \mathrm{C}_{6}$ increased the $\beta$-transition of starch and the $\alpha$ transition of PBS. This behavior would indicate an enhancement of the interfacial adhesion between the PBS and starch.

The enhanced compatibilization induced by these amphiphilic polycondensates was presumably owing to hydrophobic interactions with PBS and hydrogen bonding with starch.

Morphology. The interest of combining SEM and confocal microscopy is to first obtain information about the distribution of the compatibilizers in the complete material structure; second, the use of SEM allows access to the morphology of the material. The comparison of both measurements allows conclusions about the distribution and effects of compatibilizers in/on the material morphology.

Confocal microscopy. In this study, the morphology of the TPS-PBS blends compatibilized with poly(bis-pyrrolidone) was examined. Neither starch nor PBS exhibit fluorescent properties.

Although pyrrolidone does not belong to the organic molecules that present typical fluorescent groups, this compatibilizer nevertheless exhibits fluorescent behavior.

Different examples of fluorescent PAMAM dendrimers (poly(amido-amine)) containing amine or hydroxyl groups have been described. ${ }^{35,36}$ The fluorescence of our synthesized compounds could be explained by the presence of the same groups as in these PAMAM dendrimers.

Figure 7 shows the morphology of the blends compatibilized with $\mathrm{C}_{2} \mathrm{C}_{2}$ (SPB30 and SPB70).

As expected, $\mathrm{C}_{2} \mathrm{C}_{2}$ did not improve the compatibilization adhesion between the starch and the PBS. In fact, less fluorescence was obtained for SPB30 than for SPB70. The black and red areas depict the PBS and starch phases, respectively. This result implies that the $\mathrm{C}_{2} \mathrm{C}_{2}$ compatibilizer adhered only to the starch. However, this interaction is because of an interaction between the hydroxyl group in the starch molecule and the amide groups of the compatibilizer. This morphology suggests that the hydrophobic $-\mathrm{CH}_{2}$ - sequences in $\mathrm{C}_{2} \mathrm{C}_{2}$ are too small to allow an affinity with PBS. The fluorescence behavior of the other compatibilizers was characterized by a smaller black area than with $\mathrm{C}_{2} \mathrm{C}_{2}$. However, longer hydrophobic sequences in compatibilizers should lead to good interactions with PBS.
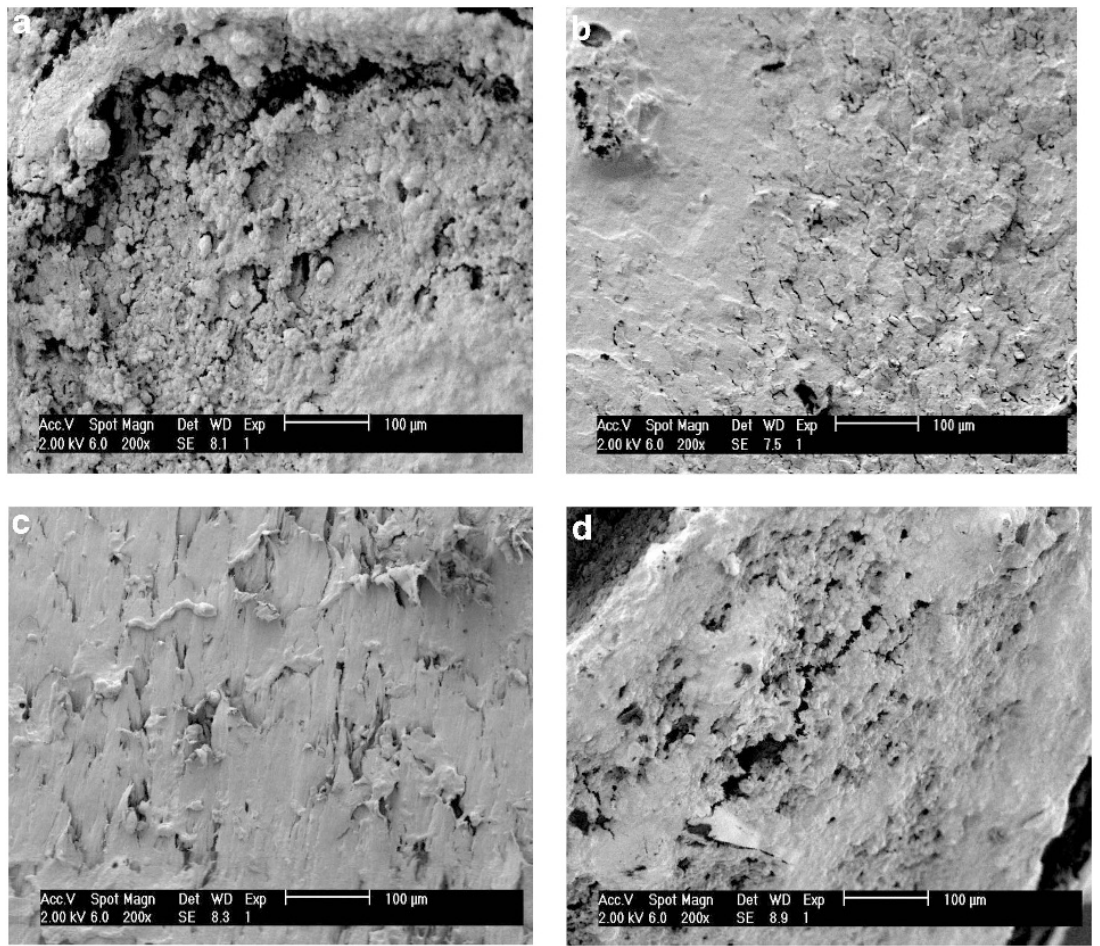

Figure 8 Effect of synthesized compatibilizers on the morphology of SPB30 using scanning electron microscopy (SEM): (a) SPB30- $\mathrm{C}_{2} \mathrm{C}_{2}$, (b) SPB30- $\mathrm{C}_{2} \mathrm{C}_{6}$ (c) SPB30- $\mathrm{C}_{6} \mathrm{C}_{2}$ and (d) SPB30- $\mathrm{C}_{6} \mathrm{C}_{6}$. 
Figure 7e shows the $\mathrm{C}_{6} \mathrm{C}_{6}$ insolubles in the SPB30- $\mathrm{C}_{6} \mathrm{C}_{6}$ blend, and confirms the visual observation: the $\mathrm{C}_{6} \mathrm{C}_{6}$ structure is also too hydrophobic to obtain an interaction with the binary system, even with the hydrophobic polyester phase.

As expected, a more homogeneous distribution is observed in Figures $7 \mathrm{c}$ and $\mathrm{d}$, which reveals that the $\mathrm{C}_{6} \mathrm{C}_{2}$ and $\mathrm{C}_{2} \mathrm{C}_{6}$ structures have a good hydrophobic-hydrophilic balance for an optimum interaction with the PBS-starch system

SEM observations. Figure 8 presents the SEM micrographs of the SPB30-compatibilizer blends. Compatibilization with $\mathrm{C}_{2} \mathrm{C}_{2}$ resulted in a further reduction of the cavities in TPS, which indicated a slight enhancement of the compatibility between the PBS and starch. Improved compatibilization morphology was also observed in the case of $\mathrm{C}_{6} \mathrm{C}_{2}$ and $\mathrm{C}_{2} \mathrm{C}_{6}$. In addition, the micrographs (Figures $8 \mathrm{~b}$ and c) revealed a homogeneous distribution of the starch phase at the investigated scale, which suggests the formation of a co-continuous phase with the polyester.

\section{DISCUSSION}

The mechanical properties of the blends of starch with PBS, as determined using extrusion processes, are poor as a result of incompatibility. Increasing the starch content decreased the modulus and tensile strength (Figure 2).

Control over the morphology of these materials is a key point of the polymer blend system but also depends on the polymer volume ratio (Figure 4). At a low-starch content, starch particles are dispersed in the PBS. When the concentration of starch increases, the dispersed starch particles form a larger continuous phase in the entire sample. In this study, the SPB30 system was chosen to be compatibilized for the following reasons: (i) SPB30 is a typical ratio for industrial polyester/ starch blends; and (ii) SPB30 shows a dispersed morphology, and this work sought to convert the SPB30 system to a co-continuous morphology, using amphiphilic compatibilizers synthesized from itaconic acid.

The transformation from a co-continuous to a dispersed phase was achieved by the use of $\mathrm{C}_{2} \mathrm{C}_{6}$ or $\mathrm{C}_{6} \mathrm{C}_{2}$ as compatibilizers. The change in morphology was observed by SEM, which revealed a better dispersion in PBS in the presence of compatibilizer (Figure 8). The increase in the interfacial surface affected the macromolecular movements, as shown in Figure 6 (a slight effect on the $\beta$ - and $\alpha$-transition temperatures). This result can be explained by hydrogen interactions between, on the one hand, amide and ester functional groups and on the other hand, amide and hydroxyl groups.

Therefore, we improved the mechanical properties of the SPB30 blend by compatibilization with an amphiphilic copolymer $\left(\mathrm{C}_{2} \mathrm{C}_{6}\right.$ and $\left.\mathrm{C}_{6} \mathrm{C}_{2}\right)$. That both molecules had the same affect reveals that the compatibilization effect is simply controlled by the hydrophilic-hydrophobic balance, regardless of the position of the functional groups. A good distribution of the compatibilizer in the polymer matrix was revealed using confocal microscopy (Figure 7).

\section{CONCLUSIONS}

Plasticized starch can be successfully blended with PBS by compatibilization with an amphiphilic copolymer. The mechanical properties of the polymer blend studied here were improved through the addition of an amphiphilic polycondensate, $\mathrm{C}_{2} \mathrm{C}_{6}$ or $\mathrm{C}_{6} \mathrm{C}_{2}$, which most likely interacts with the TPS and PBS by hydrogen bonding to the amide groups. The good distribution of the compatibilizer in the polymer matrix was demonstrated using confocal microscopy. The
$\mathrm{C}_{2} \mathrm{C}_{6}$ and $\mathrm{C}_{6} \mathrm{C}_{2}$ compatibilizers expanded the co-continuous morphology of the SPB30 samples. The change in morphology was confirmed using SEM, which revealed greater dispersion in PBS in the presence of the compatibilizer.

Amphiphilic compatibilizers could provide an interesting alternative to the frequently used reactive compatibilizers, such as maleic anhydride, which are more difficult to manipulate.

The use of itaconic acid, which is a renewable chemical identified as one of the top 12 value-added chemicals that can be obtained from biomass as one of the main components of these new compatibilizers, is another interesting feature of this study.

1 Ayadi, F. \& Dole, P. Stoichiometric interpretation of thermoplastic starch water sorption and relation to mechanical behavior. Carbohydr Polymers 84, 872-880 (2011).

2 Follain, N., Joly, C., Dole, P. \& Bliard, C. Mechanical properties of starch-based materials. I. Short review and complementary experimental analysis. J App/ Polym Sci 97, 1783-1794 (2005).

3 Avérous, L. Biodegradable multiphase systems based on plasticized starch: a review. J Macromol Sci, Part C 44, 231-274 (2004).

4 Jang, W. Y., Shin, B. Y., Lee, T. J. \& Narayan, R. Thermal properties and morphology of biodegradable PLA/starch compatibilized blends. J Ind Eng Chem 13, 457-464 (2007).

5 Li, H. \& Huneault, M. A. Comparison of sorbitol and glycerol as plasticizers for thermoplastic starch in TPS/PLA blends. J Appl Polym Sci 119, 2439-2448 (2011).

6 Arroyo, O. H., Huneault, M. A., Favis, B. D. \& Bureau, M. N. Processing and properties of PLA/thermoplastic starch/montmorillonite nanocomposites. Polym Composite 31, 114-127 (2010).

7 Dubois, P. \& Narayan, R. Biodegradable compositions by reactive processing of aliphatic polyester/polysaccharide blends. Macromol Symp 198, 233-244 (2003).

8 Chen, L., Zhang, Z., Zhuang, X., Chen, X. \& Jing, X. Compatibilizing effect of starchgrafted-poly(L-lactide) on the poly(\&-caprolactone)/starch composites. J App/ Polym Sci 117, 2724-2731 (2010).

$9 \mathrm{Li}$, G. \& Favis, B. D. Morphology development and interfacial interactions in polycaprolactone/thermoplastic-starch blends. Macromol Chem Physics 211, 321-333 (2010).

10 Mariani, P. D. S. C., Allganer, K., Oliveira, F. B., Cardoso, E. J. B. N. \& Innocentini-Mei, L. H. Effect of soy protein isolate on the thermal, mechanical and morphological properties of poly ([var epsilon]-caprolactone) and corn starch blends. Polym Test $\mathbf{2 8}$ 824-829 (2009).

11 Zeng, J. B., Jiao, L., Li, Y. D., Srinivasan, M., Li, T. \& Wang, Y. Z. Bio-based blends of starch and poly(butylene succinate) with improved miscibility, mechanical properties, and reduced water absorption. Carbohydr Polymers 83, 762-768 (2011).

12 Flores, D. E., Funabashi, M. \& Kunioka. Mechanical Properties and Biomass Carbon Ratios of Poly(butylene succinate) Composites Filled with Starch and Cellulose Filler Using Furfural as Plasticizer. Vol. 112 (Wiley, 2009)

13 Dean, K., Yu, L., Bateman, S. \& Wu, D. Y. Gelatinized starch/biodegradable polyester blends: processing, morphology, and properties. J App/ Polym Sci 103, 802-811 (2007).

14 Briassoulis, D. An overview on the mechanical behaviour of biodegradable agricultural films. J Polym Environ 12, 65-81 (2004).

15 Landreau, E., Tighzert, L., Bliard, C., Berzin, F. \& Lacoste, C. Morphologies and properties of plasticized starch/polyamide compatibilized blends. Eur Polym J 45, 2609-2618 (2009).

16 Ramkumar, D. \& Bhattacharya, M. Effect of crystallinity on the mechanical properties of starch/synthetic polymer blends. J Mater Sci 32, 2565-2572 (1997).

17 Mani, R. \& Bhattacharya, M. Properties of injection moulded blends of starch and modified biodegradable polyesters. Eur Polym J 37, 515-526 (2001).

18 Willke, T. \& Vorlop, K. D. Biotechnological production of itaconic acid. App/ Microbiol Biotechnol 56, 289-295 (2001).

19 Werpy, T., Holladay, J. \& White, J. Top Value Added Chemicals From Biomass: I. Results of Screening for Potential Candidates from Sugars and Synthesis Gas (DOE Scientific and Technical Information, 2004).

20 Paytash, P. L., Thompson, M. J. \& Fykes, M. E. Itaconic acid derivatives of sulfanilamide. J Am Chem Soc 74, 4549-4552 (1952).

21 Lourdin, D., Coignard, L., Bizot, H. \& Colonna, P. Influence of equilibrium relative humidity and plasticizer concentration on the water content and glass transition of starch materials. Polymer (Guildf) 38, 5401-5406 (1997).

22 Onteniente, J. P., Etienne, F., Bureau, G. \& Claude, J. Fully biodegradable lubricated thermoplastic starches: water desorption on extruded samples. Starch-Stärke 48, 10-16 (1996).

23 Makal, U. \& Wynne, K. J. Water induced hydrophobic surface. Langmuir 21, 3742-3745 (2005)

24 Averous, L. \& Boquillon, N. Biocomposites based on plasticized starch: thermal and mechanical behaviours. Carbohydr Polymers 56, 111-122 (2004).

25 Averous, L., Moro, L., Dole, P. \& Fringant, C. Properties of thermoplastic blends: starch-polycaprolactone. Polymer (Guildf) 41, 4157-4167 (2000). 
26 Schwach, E. \& Avérous, L. Starch-based biodegradable blends: morphology and interface properties. Polym Int 53, 2115-2124 (2004).

27 Averous, L., Fauconnier, N., Moro, L. \& Fringant, C. Blends of thermoplastic starch and polyesteramide: processing and properties. J App/ Polym Sci 76, 1117-1128 (2000).

$28 \mathrm{Kim}, \mathrm{H} .$, Hee, S. Y., Han, S. \& Hyun, J. Biodegradability and Mechanical Properties of Agro-Flour- Filled Polybutylene Succinate Biocomposites Vol. 97 (Wiley, 2005).

29 Bhatia, A., Gupta, R. K., Bhattacharya, S. N. \& Choi, H. J. Compatibility of biodegradable poly (lactic acid) (PLA) and poly (butylene succinate) (PBS) blends for packaging application. Korea-Aust Rheol J 19, 125-131 (2007).

30 Martin, O. \& Avérous, L. Poly(lactic acid): plasticization and properties of biodegradable multiphase systems. Polymer (Guildf) 42, 6209-6219 (2001).

31 Wang, R., Wang, S. \& Zhang, Y. Morphology, Mechanical Properties, and Thermal Stability of Poly(L-lactic acid)/Poly(butylene succinate-co-adipate)/ Silicon Dioxide Composites. Vol. 113 (Wiley, 2009).
32 Lourdin, D., Bizot, H. \& Colonna, P. 'Antiplasticization' in starch-glycerol films? J Appl Polym Sci 63, 1047-1053 (1997).

33 Forssell, P. M., Mikkilä, J. M., Moates, G. K. \& Parker, R. Phase and glass transition behaviour of concentrated barley starch-glycerol-water mixtures, a model for thermoplastic starch. Carbohydr Polymers 34, 275-282 (1997).

34 Averous, L. \& Fringant, C. Association between plasticized starch and polyesters: processing and performances of injected biodegradable systems. Polym Eng Sci 41, 727-734 (2001).

35 Wang, D., Imae, T. \& Miki, M. Fluorescence emission from PAMAM and PP dendrimers. J Colloid Interface Sci 306, 222-227 (2007).

36 Porcar, L., Liu, Y., Verduzco, R., Hong, K., Butler, P. D., Magid, L. J., Smith, G. S. \& Chen, W. R. Structural investigation of PAMAM dendrimers in aqueous solutions using small-angle neutron scattering: effect of generation. J Phys Chem B 112, 14772-14778 (2008). 